\title{
Control of Airborne Microorganisms by Essential Oils Released by VaxiPod
}

\author{
Ekaterina Mirskaya and Igor E. Agranovski * \\ School of Engineering and Built Environment, Griffith University, Brisbane, QLD 4111, Australia; \\ kate.mirskaya@griffith.edu.au \\ * Correspondence: i.agranovski@griffith.edu.au; Tel.: +617-3735-7923
}

Citation: Mirskaya, E.;

Agranovski, I.E. Control of Airborne Microorganisms by Essential Oils Released by VaxiPod. Atmosphere 2021, 12, 1418. https://doi.org/ $10.3390 /$ atmos 12111418

Academic Editor: Salvatore Romano

Received: 8 October 2021

Accepted: 25 October 2021

Published: 28 October 2021

Publisher's Note: MDPI stays neutral with regard to jurisdictional claims in published maps and institutional affiliations.

Copyright: (c) 2021 by the authors. Licensee MDPI, Basel, Switzerland. This article is an open access article distributed under the terms and conditions of the Creative Commons Attribution (CC BY) license (https:// creativecommons.org/licenses/by/ $4.0 /)$.

\begin{abstract}
Currently, due to the global pandemic caused by severe acute respiratory syndrome coronavirus SARS-CoV-2, new procedures and devices for effective disinfection of indoor air are of obvious interest. Various studies demonstrated quite broad ranges of the efficiency of essential oils in the control of biological aerosols. This project reports the results of investigation of the antimicrobial activity of essential oils natural for Australia (tea tree oil, eucalyptus oil and lemon myrtle) distributed by newly developed VaxiPod device for various scenarios, including bacterial, viral and fungal inactivation on various surfaces and in aerosol form. It was found that the device was capable of operating continuously over 24 -h periods, providing sufficient aerosol concentration to efficiently inactivate microorganisms both on the surface and in airborne form. Twenty-four to forty-eight hours were required to achieve inactivation above $90 \%$ of most of the tested microbes on solid surfaces (stainless steel discs and agar plates), whilst similar efficiency of inactivation on fibrous filter surface as well as in aerosol form was achieved over 30-60 min of the process run. The results look very promising for further development of bioaerosol inactivating procedures and technologies for air quality control applications.
\end{abstract}

Keywords: bioaerosol control; fungal spores; bacteria; virus; essential oils; microbial inactivation

\section{Introduction}

In the global pandemic situation, the prevention and mitigation of pathogenic microorganisms in indoor air spaces and on various surfaces attract significant attention. While conventional disinfection methods may be limited by reliance on the operator, the use of 'no-touch' automated disinfection systems provides a promising alternative [1]. The disinfectants used for these systems should not only be effective in reducing the microorganisms present in the air but should also not be toxic for humans, allowing their continuous application.

Essential oils (EOs) are complex mixtures of volatile liquid and lipophilic compounds obtained by parts of plants by extraction, effleurage, drag steam vapour, extraction with organic solvents, pressing, or supercritical fluid extraction [2]. EOs have been shown to have bactericidal, fungicidal, virucidal, and phagocidal activities. The antimicrobial effects of EOs are explained by their composition and cytotoxic effects, which cause cell membrane damage.

Australian endemic plants produce a variety of essential oils such as tea tree oil (oil of Melaleuca alternifolia), eucalyptus oil (oil of Eucalyptus polybractea) and lemon myrtle oil (oil of Backhousia citriodora) which are used in health products, aromatherapy, perfumes, bushfoods, food flavourings and herbal teas [3].

The potential use of Melaleuca alternifolia (Tea tree) oil as a disinfectant has been clearly shown in the literature for treating bacteria $[4-6]$, fungi $[7,8]$ and viruses $[9,10]$. The antimicrobial activity of Tea Tree Oil (TTO) is attributed mainly to terpinen-4-ol (35-45\%) and 1,8-cineole (1-6\%); however, other components such as a-terpineol, terpinolene and a- and c-terpinene are also often present and potentially contribute to microbial disinfection [11]. 
Eucalyptus oil obtained from Eucalyptus corresponds to one of the principal genera of the Myrtaceae family. Eucalyptus oil is usually rich in monoterpenes and, in some cases, sesquiterpenes. Eucalyptus oil was evaluated against several Gram-positive and Gram-negative bacterial strains as well as against various fungal and viral species and showed different degrees of efficiency [12,13]. Both tea tree oil and eucalyptus oil were challenged as aerosols and vapours and demonstrated very promising capabilities on the inactivation of pathogenic airborne microbes over short periods of time [14].

The major component of Backhousia citriodora oil is citral $(>85 \%)$, with two main isomeric aldehydes: neral and geranial with minor amounts of (E)- $\beta$-ocimene, $p$-cymene, citronellal, linalool, $\beta$-elemene, $\beta$-caryophyllene, nerol, geraniol, globulol and spathulenol [15]. Lemon myrtle oil was shown to possess significant antimicrobial activity against bacteria, including Escherichia coli and methicillin-resistant S. aureus, and fungi including Aspergillus niger [16]. The antimicrobial activity of Lemon myrtle EO was found to be greater than that of citral alone and often superior to Melaleuca alternifolia essential oil [17].

A number of studies reported a greater antimicrobial effect of EO vapour compared with EOs in liquid form applied by direct contact $[18,19]$. The use of combinations of EOs is capable of increasing the efficacy of EOs, taking advantage of their synergistic and additive effects [20]. Although various approaches for the evaluation of microbial inactivation by EOs in the vapour phase are provided in the literature, no standard assay has been developed.

The growth of methicillin-resistant Staphylococcus aureus (MRSA) on seeded agar plates was reduced by $38 \%$ after $20 \mathrm{~h}$ of exposure to a blend of geranium and lemongrass Eos, dispersed via a generator that releases vapours into the air by means of negative and Venturi airflow in a sealed box environment. In an office environment, the same disinfection method achieved an $89 \%$ reduction of airborne bacteria in $15 \mathrm{~h}$ when operated at a constant output of $100 \%$ [21]. A significant reduction in viable numbers of $E$. coli and S. aureus inoculated on agar plates was obtained when the bacteria were exposed to a flame of candles containing orange oil in a large air-tight chamber for $3 \mathrm{~h}$. As the aerial concentration of the volatiles was increased, the viability of E. coli and S. aureus declined [22].

Inouye et al. [19] showed that the rapid evaporation of essential oils is more effective than slow evaporation in a small $(1.3 \mathrm{~L})$ enclosure. Antibacterial activity of tea tree, eucalyptus and lemongrass oils by gaseous contact against $E$. coli was achieved at the minimal inhibitory dose (MIC) of 50, >1600 and $100 \mathrm{mg} / \mathrm{L}$ air, respectively. These high concentrations seem hardly achievable with a domestic oil diffusor.

Chao et al. [23] investigated the antibacterial activity of an oil blend including cinnamon, clove, rosemary, eucalyptus and lemon EOs against bacterial bioaerosols into a $0.4 \mathrm{~m}^{3}$ enclosed fume hood. An $82 \%$ reduction in M. luteus bioaerosol, a $96 \%$ reduction in the $P$. aeruginosa bioaerosol and a $44 \%$ reduction in the $S$. aureus bioaerosol were detected following $10 \mathrm{~min}$ of exposure.

The dispersion of a mixture of Citrus limon EO and Abies alba EO using commercially available ultrasonic vaporisers in hospital rooms resulted in a $40 \%$ reduction in bacteria, and a 30-60\% reduction in fungal contamination was observed in the first two hours [24]. In another study, terpineol, an important compound of tea tree oil, showed an average reduction of the germ count in a testing room of $59.4 \%$ after $5 \mathrm{~h}$ of spreading when vaporised with a room diffuser [25].

Reductions in both bacterial and fungal contamination were observed between rooms cleaned using standard sanitisation alone or in combination with a mixture of essential oils containing Lavender, Cajeput, Siberian Fir, Myrtle and Geranium bourbon EOs dispersed using commercially available ultrasound vaporisers in a residential health care house [26]. The potential reduction of bacterial and fungal contamination in unventilated lab spaces and unventilated real cultural heritage assets when tea tree and thyme EOs are cold diffused over $24 \mathrm{~h}$ has recently been evaluated. Tea tree EO vaporisation showed an up to $77.3 \%$ fungal and $95.0 \%$ bacterial air contamination reduction [27]. Lavender essential oil 
dispersed by automatic diffusers significantly reduced the number of bacteria in hospital areas [28].

Evaluation of the antiviral activity of tea tree and eucalyptus EOs aerosolised via a nebuliser showed strong virucidal activity against airborne influenza virus A strain [13] and phagocidal activity against E. coli phage M13 [14].

The aim of this study is the determination of antimicrobial activities of a mixture of Melaleuca alternifolia, Eucalyptus polybractea and Backhousia citriodora essential oils dispersed via a novel oil atomizer VaxiPod (Model: \#ALNEBVP01; Linfield Pacific P/L, Gladesville, NSW, Australia). The EO blend was formulated, taking into account the chemical composition and specific activity of each. The antimicrobial activity of the oil blend against two bacterial (Escherichia coli and Bacillus subtilis), one fungal (Aspergillus niger) and one viral (bacteriophage MS2) strain was considered on a porous and nonporous surface as well as in aerosolised form.

\section{Materials and Methods}

\subsection{Microorganisms}

The working culture of E. coli was prepared from the stock culture on nutrient agar (NA) and incubated at $37^{\circ} \mathrm{C}$. After $24 \mathrm{~h}$, the culture was aseptically transferred to $10 \mathrm{~mL}$ of nutrient broth and incubated for $19 \mathrm{~h}$ at $37^{\circ} \mathrm{C}$. Cells were washed twice by centrifugation in sterile distilled water. The resulting pellet was resuspended in sterile Phosphate-Buffered Saline (PBS), and the number of cells in the suspension was adjusted to achieve a number of colony-forming units (CFU) between $10^{7}$ and $10^{9} \mathrm{CFU} / \mathrm{mL}$. The working culture of B. subtilis was prepared in the same way.

The working culture of Aspergillus niger was prepared from the stock culture on malt extract agar (MEA) and incubated at $25^{\circ} \mathrm{C}$. After $48 \mathrm{~h}$, a second subculture was prepared in the same way and grown for 7 days. A bowl with water was placed in the incubator to keep the relative humidity at $55-65 \% .10 \mathrm{~mL}$ of sterile PBS was placed on the surface of the working culture. The conidiospores were disclosed using a sterile glass spatula and the suspension was transferred into a conical flask and shaken gently for $1 \mathrm{~min}$. The number of spores in suspension was adjusted to achieve the number of CFU between $10^{6}$ and $10^{7} \mathrm{CFU} / \mathrm{mL}$.

MS2 bacteriophage was recovered from a freeze-dried state and propagated on plates with soft-agar/host overlay. Host strain of E. coli was cultured in 2YT medium broth (Bacto tryptone $16 \mathrm{~g}$; yeast extract $10 \mathrm{~g}$; $\mathrm{NaCl} 5 \mathrm{~g}$; distilled water $1 \mathrm{~L}$ ). To prepare viral stock, a log-stage host cells culture was infected with phage from a single plaque and incubated overnight at $37^{\circ} \mathrm{C}$ with constant agitation. Overnight phage culture was centrifuged twice at high speed to remove cells and then chloroform to prevent microbial growth was added. Decimal dilutions of phage stock were made in PBS to achieve the number of plaque-forming units (PFU) between $10^{8}$ and $10^{9} \mathrm{PFU} / \mathrm{mL}$.

All preparations and experiments were conducted in a Class II biological safety cabinet (Model BH 2000, Biolab, Australia) to prevent microbial release into the laboratory environment.

\subsection{Essential Oil and VaxiPod Atomiser}

Melaleuca alternifolia (Tea tree), Eucalyptus polybractea (Eucalyptus) and Backhousia citriodora (Lemon myrtle) EOs acquired from Bosisto's and NATIO (Australia) and produced by Australian endemic plants were used for the experiments. The EO blend was formulated, taking into account the chemical composition and specific activity of each and included a mixture of Tea tree, Eucalyptus and Lemon myrtle in ratio 4.5:4.5:1. This ratio has been proposed to expand the range of microbes that are affected by different, but not overlapping, chemical constituents of the oils. In addition, the Lemon Myrtle also served to impart a pleasant scent to the oil mixture. The oil blend was dispersed via the VaxiPod oil atomiser, providing rate of the oil mixture release of $\sim 1.0 \mathrm{~mL} / \mathrm{h}$. 


\subsection{Inactivation of Microorganisms on Porous Filter Surface}

The experimental setup used for this part of investigation is presented in Figure 1. Such arrangement (four parallel filters) was convenient for direct comparison of a timerelated microbial decay results within each particular run, as all initial experimental conditions, including number of microbes on the surface as well as physical parameters of the airflow, were identical. Sterile polypropylene fibre filters were used as carriers in this investigation. The carriers were placed in sterile Petri dishes and contaminated by depositing $0.05 \mathrm{~mL}=50 \mu \mathrm{L}$ of microbial suspension in the centre of the carrier. The inoculum was spread evenly on the carrier, covering the area of $3.0 \pm 0.5 \mathrm{~cm}^{2}$. Then, the carriers were placed in the filter holder for experimental use.

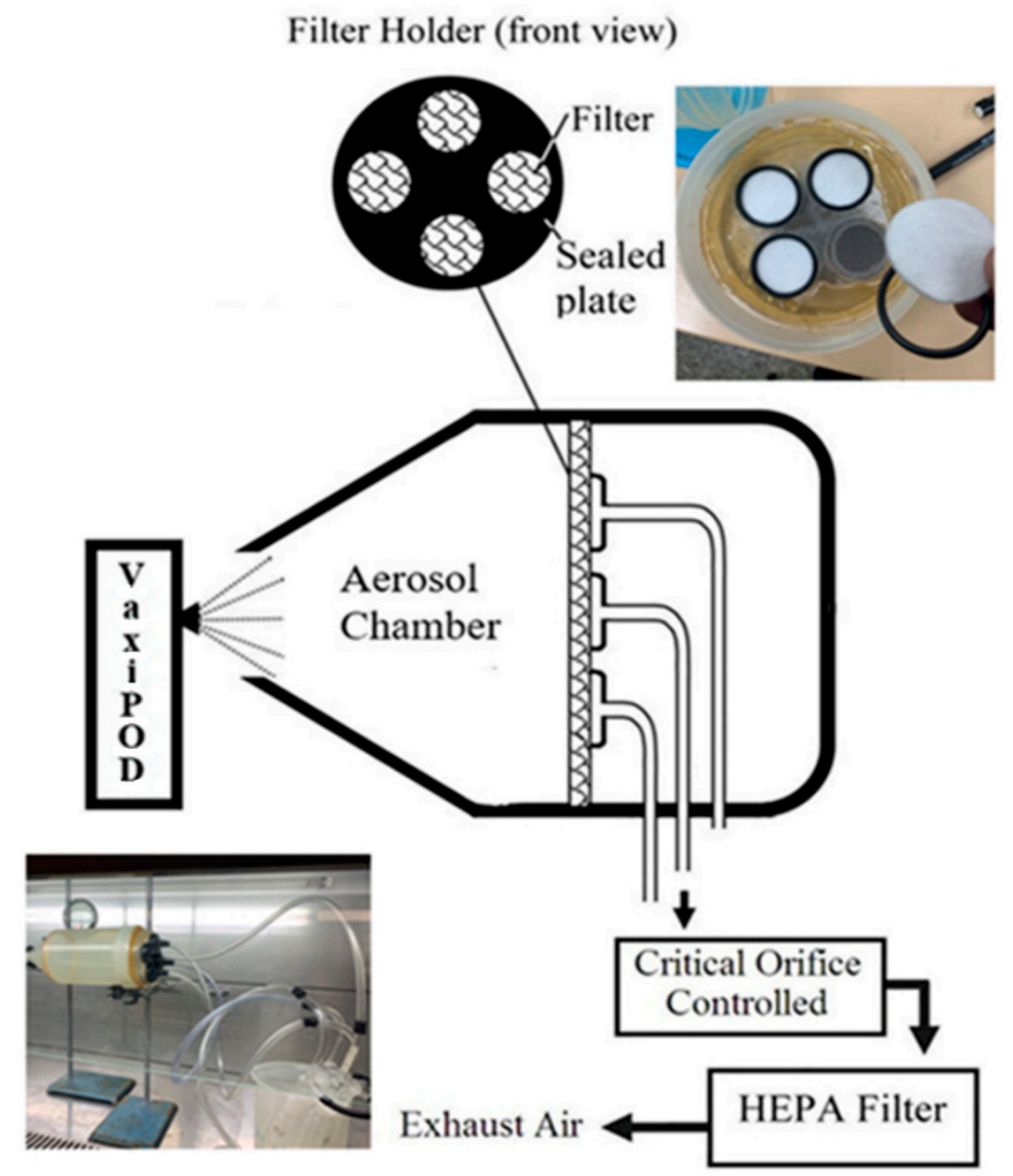

Figure 1. Microbial inactivation on filter surface-laboratory setup.

Two experimental runs were undertaken for each microorganism used in this work. The first run did not involve any disinfecting substances that can have an impact on the survivability of test microorganisms (Vaxipod was not operating and no oils were added to the air carrier); only natural microbial inactivation on the filter surface resulting from interaction with the airflow was evaluated. In the second part of the experiment, the oil atomiser, containing a mixture of tea tree oil, eucalyptus oil and lemon myrtle, was placed at the entry of the aerosol chamber. After passing through the filter stage, the stream was HEPA-filtered and discharged to the atmosphere. The temperature and RH were kept at levels of $25^{\circ} \mathrm{C}$ and 50 to $55 \%$, respectively, throughout all experimental runs. 
The following procedure was used for all experimental runs. A filter holder with four filters contaminated with inoculum was stationary fixed and hermetically sealed in the chamber to avoid any possible air bypass. For the first part of the experiment, the filters were removed after at 15-, 30-, 60- and 120-minute intervals of the sampling pump operation. Then, another four filters similarly contaminated with inoculum were placed in the filter holder, stationary fixed and hermetically sealed in the chamber, and the oil atomiser was switched on. The filters were removed at 15-, 30-, 60- and 120-minute intervals. Immediately upon removal from the holder, each filter was placed into the glass bottle containing $50 \mathrm{~mL}$ of sterile PBS and sonicated in a sonic bath (Biolab, Clayton, VIC, Australia) for a period of $1 \mathrm{~min}$ to ensure that the majority of particles were transmitted from the filter to the liquid.

Then, an aliquot of $0.1 \mathrm{~mL}$ of an appropriate 10-fold dilution of the liquid was spread on the surface of the NA for E. coli and B. sublilis and MEA medium for A. niger. NA plates were placed in an incubator set at $37^{\circ} \mathrm{C}$ for $24 \mathrm{~h}$, MEA plates were grown at $25^{\circ} \mathrm{C}$ for 3-7 days and CFUs were counted after incubation. Concentration of live phage particles was determined by a double-agar-layer plaque technique [29]. A hard agar medium was prepared from 2YT medium supplemented with $2 \%$ agar and a soft agar medium with $0.5 \%$ agar. The results were expressed in Plaque-Forming Units (PFU) per millilitre of suspension.

\subsection{Inactivation of Microorganisms on Nonporous Stainless Steel Surface}

Sterile 301 stainless steel discs of $4 \mathrm{~cm}$ in diameter and surface-finished on both sides were placed in sterile Petri dishes and inoculated with $50 \mu \mathrm{L}$ of microbial suspension. The inoculum was spread evenly on the carrier, covering an area of $3.0 \pm 0.5 \mathrm{~cm}^{2}$. The carriers were allowed to dry in an incubator at $37^{\circ} \mathrm{C}$ for $20 \mathrm{~min}$. For each test organism, two identical carriers were contaminated for survival control. Control carriers were prepared in the same way but not exposed to the aerosolised oil mixture and kept outside the test enclosures during the experimental runs.

For all the experiments, the test enclosure $\left(0.7 \mathrm{~m}^{3}\right.$ vapour chamber $)$ was sealed to allow the oil mixture to be safely diffused inside and prevent dispersed oil escape. The enclosure was thermally insulated and homogeneous temperature $\left(25 \pm 2{ }^{\circ} \mathrm{C}\right)$ and humidity $(55 \%)$ were maintained in the enclosures during all experimental runs. The oil atomisers (evaporating rate $\sim 1.0 \mathrm{~mL} / \mathrm{h}$ ) were placed in the enclosure to continuously dispense the oil blend.

Contaminated stainless steel discs were then placed in the enclosure and exposed to the oil mixture dispersed via the oil atomiser for periods up to $48 \mathrm{~h}$. After exposure, the discs were placed in beakers containing 5 g glass beads and $10 \mathrm{~mL}$ of phosphatebuffered saline (PBS) and shaken for $1 \mathrm{~min}$ at $150 \mathrm{rpm}$ on a rotary shaker. The microbial suspensions were appropriately diluted, plated on to appropriate agar media and grown as described above. Reduction in viability (\%) was calculated based on the reduction in colony (plaque) count in the dispersed oil mixture exposed discs with respect to the control (unexposed) discs.

$$
M S t=\frac{C F U_{t}}{C F U_{0}}
$$

where MSt is the microbial survival at time $t, C F U_{t}$ is the number of live microorganisms recovered from the disc exposed to the dispersed oil mixture and $C F U_{0}$ is the number of live microorganisms recovered from the control discs.

\subsection{Inactivation of Microorganisms on Agar Surface}

It is also important to assess any potential impact of the presence of nutrients on surfaces that may affect the survival of microorganisms. Several experiments were undertaken to evaluate this scenario. They were conducted in the enclosure for selected microorganisms, including E. coli, B. subtilis and A. niger. Appropriate serial dilution of the bacterial and fungal cultures (to obtain 100-300 CFU) was plated on NA/MEA plates. 
Inoculated plates were opened inside the enclosures and placed on the bottom part of the enclosures for exposure to the essential oil mixture. To examine the long-term growth inhibitory effect of the oil mixture supplied by the oil atomiser, one set of plates with each type of microorganisms was removed from the enclosure in $0,15,24$ and $48 \mathrm{~h}$. The plates were incubated at $37^{\circ} \mathrm{C}$ for $24 \mathrm{~h}$ for bacteria and $25^{\circ} \mathrm{C}$ for $3-7$ days for fungi, and CFUs were counted after incubation. Control plates were stored at room temperature and then collected, incubated and counted in the same way. Reduction in viability (\%) was calculated based on the reduction in colony count on the oil vapour exposed plates with respect to the control plates (Equation (1)).

\subsection{Antimicrobial Activity against Biological Aerosols}

These experiments were focused on evaluation of activity of the oil mixture dispersed via oil atomiser against airborne microbes in the ambient air. The laboratory setup used for this series of experiments is shown in Figure 2. A rotational toroid shaped 200 litres aluminium aerosol chamber ( $650 \mathrm{~mm}$ diameter and $600 \mathrm{~mm}$ long) was designed to keep materials in airborne form over extended periods of time [13]. The optimal rotation speed was found to be around $7 \mathrm{rpm}$. The axis was designed to be stationary, enabling to host pipelines used for aerosol charging and monitoring.

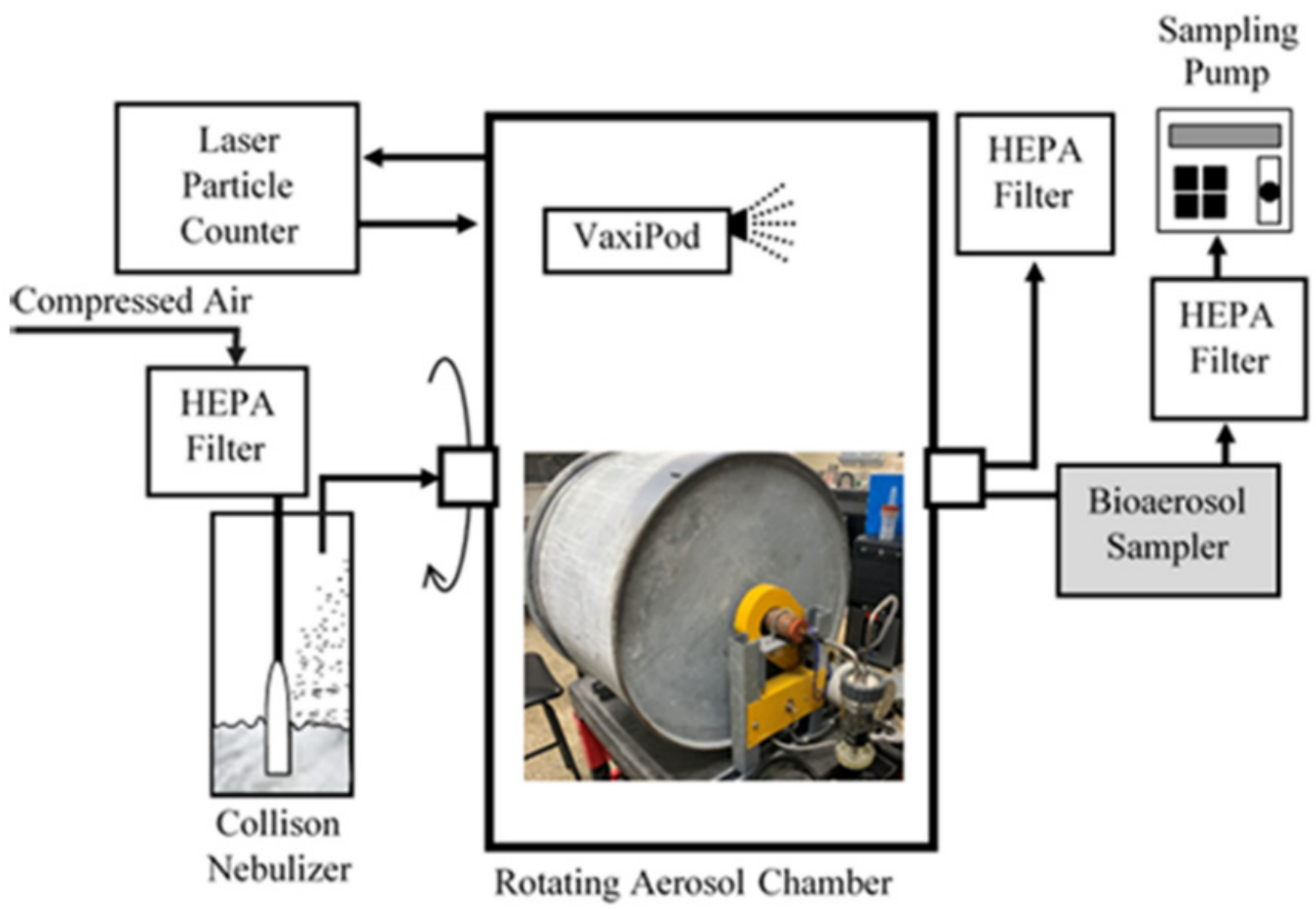

Figure 2. Inactivation of biological aerosols-Experimental setup.

The oil blend was dispersed via the oil atomiser strategically placed on a stationary shelf installed inside a rotating aerosol chamber (Figure 3). Bacterial and phage aerosols were generated by a Collison nebuliser from a liquid suspension, prepared according to the procedures described above. The microbial loading time for all experiments was $2 \mathrm{~min}$. The nebuliser operated at a flow rate of $6 \mathrm{~L} / \mathrm{min}$ of dry and filtered compressed air. 


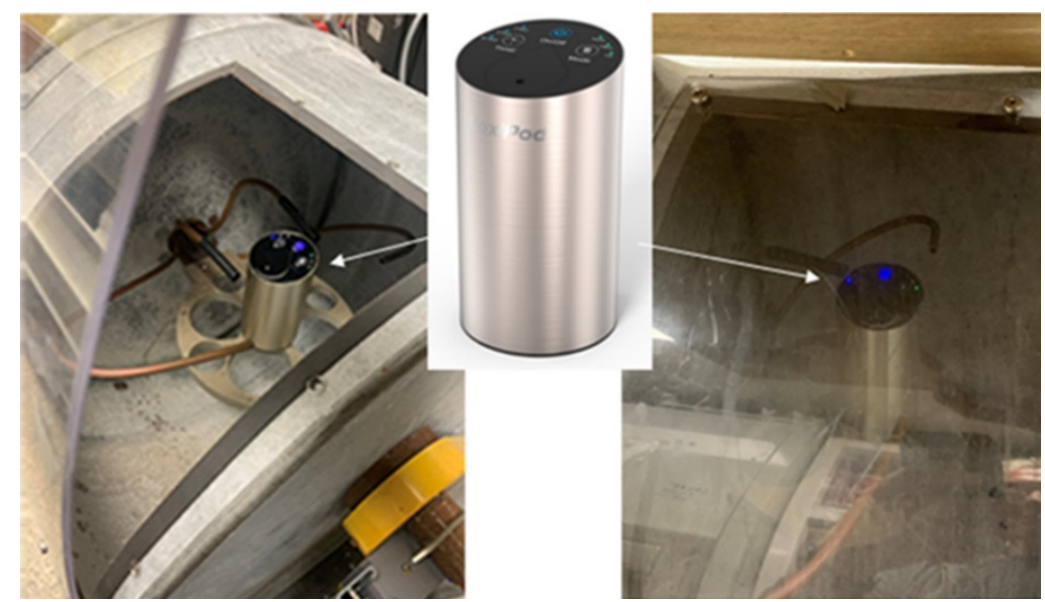

Figure 3. Location of oil atomiser inside rotational aerosol chamber.

Fungal aerosols were generated by our earlier developed device (Figure 4) based on aerosolisation of fungal spores from cultures grown in standard 9-centimetre Petri dishes by vibration [30]. In brief, a 10-centimetre diameter, 2 Watts, 4 Ohm speaker was hermetically sealed using soft rubber gasket to the bottom of cylindrical casing of the device. A Petri dish, containing mature fungal spores, was placed directly on the speaker's surface and secured with thin flexible rubber strips. The speaker size was chosen to exactly accommodate a standard 9-centimetre diameter Petri dish that is placed on it so that the Petri dish vibrated simultaneously with the speaker's membrane at different frequencies when running the experiment. A Hewlett-Packard 3312A Function Generator was used to set the amplitude and frequency of the vibrating speaker membrane to achieve the maximum possible particle concentration. A fresh dish with the fungal culture was used for each experimental run.
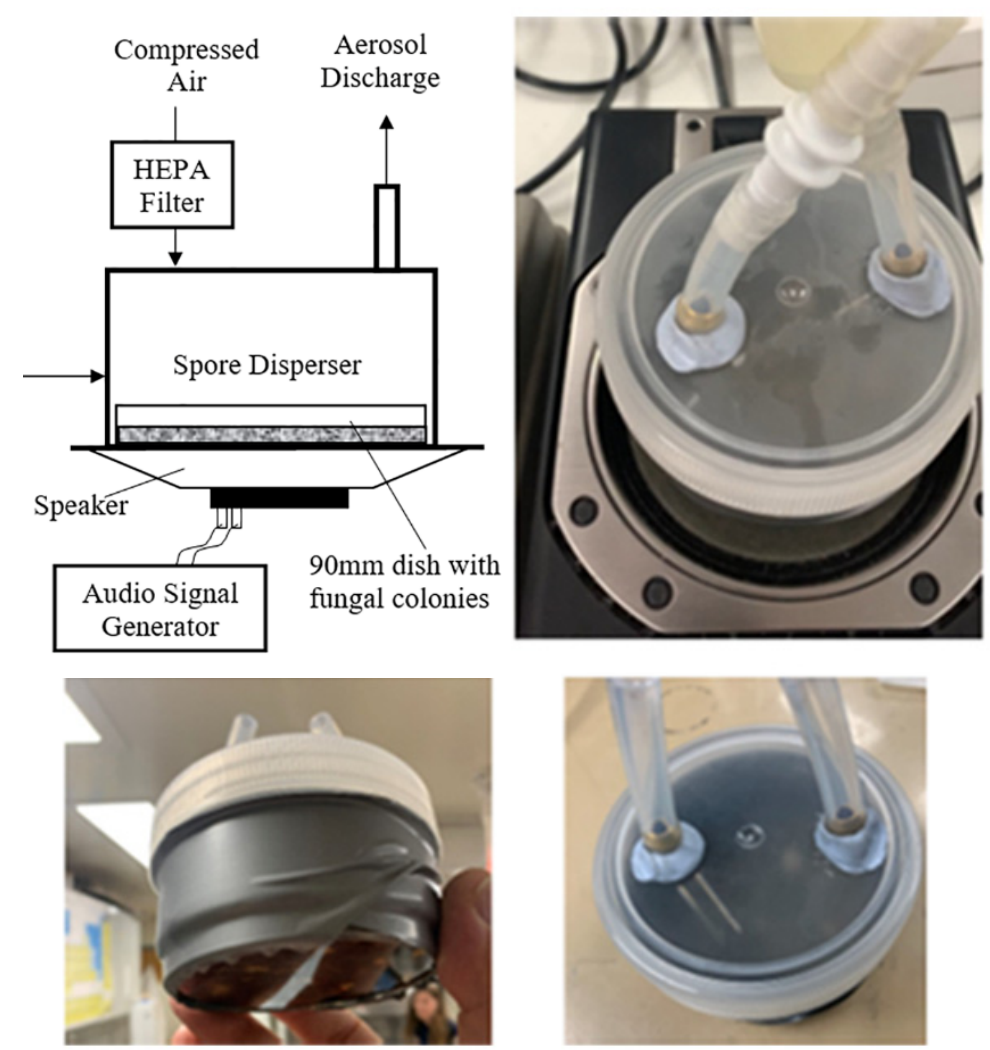

Figure 4. Fungal particle generator. 
To trace the microbial concentration in the chamber throughout the entire experiment, a fluorescent dye (fluorescein sodium salt, C20H10Na2O2, Fluka AG, Buchs, Switzerland) at concentration of $0.5 \mathrm{mg} / \mathrm{mL}$ was added into microbial suspension (see details of the procedure in Agranovski et al. [31] and Pyankov et al. [32]. Experimental conditions such as the air temperature and humidity were continuously monitored and controlled throughout the entire procedure $\left(\mathrm{T}=25^{\circ} \mathrm{C}\right.$ and $\left.\mathrm{RH}=55 \%\right)$, and uniformity of particle concentration and size distribution in the aerosol chamber were continuously monitored by a real-time Laser Aerosol Spectrometer (LAS, Model 4705, Aeronanotech, Moscow, Russia) to trace any unfavourable variations in aerosol supply.

The aerosol samples were collected after 0,10, 30, 60 and $120 \mathrm{~min}$ for E. coli, B. subtilis and $0,30,60,120$ and $200 \mathrm{~min}$ for $A$. niger at the sample flow rate of $28.3 \mathrm{~L} / \mathrm{min}$ by a Single-Stage Viable Bioaerosol Impactor (Cat. No. 225-9611, SKC, Eighty Four, PA, USA) over $12 \mathrm{~s}$ of sampling time. Upon completion of sampling, plates were removed from the impactor and placed in an incubator set at $37^{\circ} \mathrm{C}$ for $24 \mathrm{~h}$ for bacteria, whilst $A$. niger was grown at $25^{\circ} \mathrm{C}$ for $3-7$ days. On completion, the CFUs were counted and the results were calculated accordingly.

The MS2 aerosol samples were collected by "bubbler" type personal bioaerosol samplers [31,33] over 1.5 min of sampling time. Sampler's flow rate was $4 \mathrm{~L}$ of air/min. Sampling was undertaken at 15-, 30-, 60- and 120-minute time periods. Each sampler was filled with $40 \mathrm{~mL}$ of sampling liquid. Aliquots of collecting liquid $(100 \mu \mathrm{L})$ from the samplers were taken for plaque assay and fluorescence measurements, enabling the identification of microbial decay as a function of time of essential oils exposure. All results were corrected to account for the sampling-related aerosol dilution, as previously described [13].

Considering that at each sampling point, approximately $6 \mathrm{~L}$ of air $(5.7 \mathrm{~L}$ by the impactor and $6 \mathrm{~L}$ by the sampler) were removed from the chamber $(200 \mathrm{~L})$ and replaced with HEPA-filtered air of the same quantity, the following equation was used to correct each particular result to account for such dilution:

$$
C_{t}=C_{M t} \times 1.031^{n}
$$

where $C_{t}$ is corrected microbial concentration at time $t, C_{M t}$ is the actual measured microbial concentration at time $\mathrm{t}$ and $n$ is the time-related point number (at $5 \mathrm{~min}, n=0$; at $30 \mathrm{~min}$, $n=1$; etc.).

\section{Results}

\subsection{Vaxipod Performance}

A very important first step of the investigation was focused on characterisation of the particle size of droplets produced by the VaxiPod. Two instruments were used to acquire results: Malvern Spraytec (Malvern Panalytical, Malvern, UK) and LAS, as mentioned above. The main reason for using two different instruments was based on the fact that entirely different physical principles are used for their operation, making the outcomes more reliable in case of their coincidence. Such necessity was dictated by the liquid nature of the particles produced, making them quite unstable and hard to characterise. The results obtained by the Spraytec are shown at the top of Figure 5. As is seen, most of the particles are in the size range below $10 \mu \mathrm{m}$; however, some very large aerosols in the order of $100 \mathrm{~s}$ of micrometres are also present in the stream. These outcomes are in excellent agreement with the results obtained by the LAS, which are provided at the bottom of Figure 5. Of course, considering the fact that the instrument is capable to measure aerosols in the range between 0.2 and $10 \mu \mathrm{m}$, no particle in the range of $100 \mathrm{~s}$ micrometres could be detected. Nevertheless, the coincidence of the results is excellent with the pick size at around $2 \mu \mathrm{m}$. 


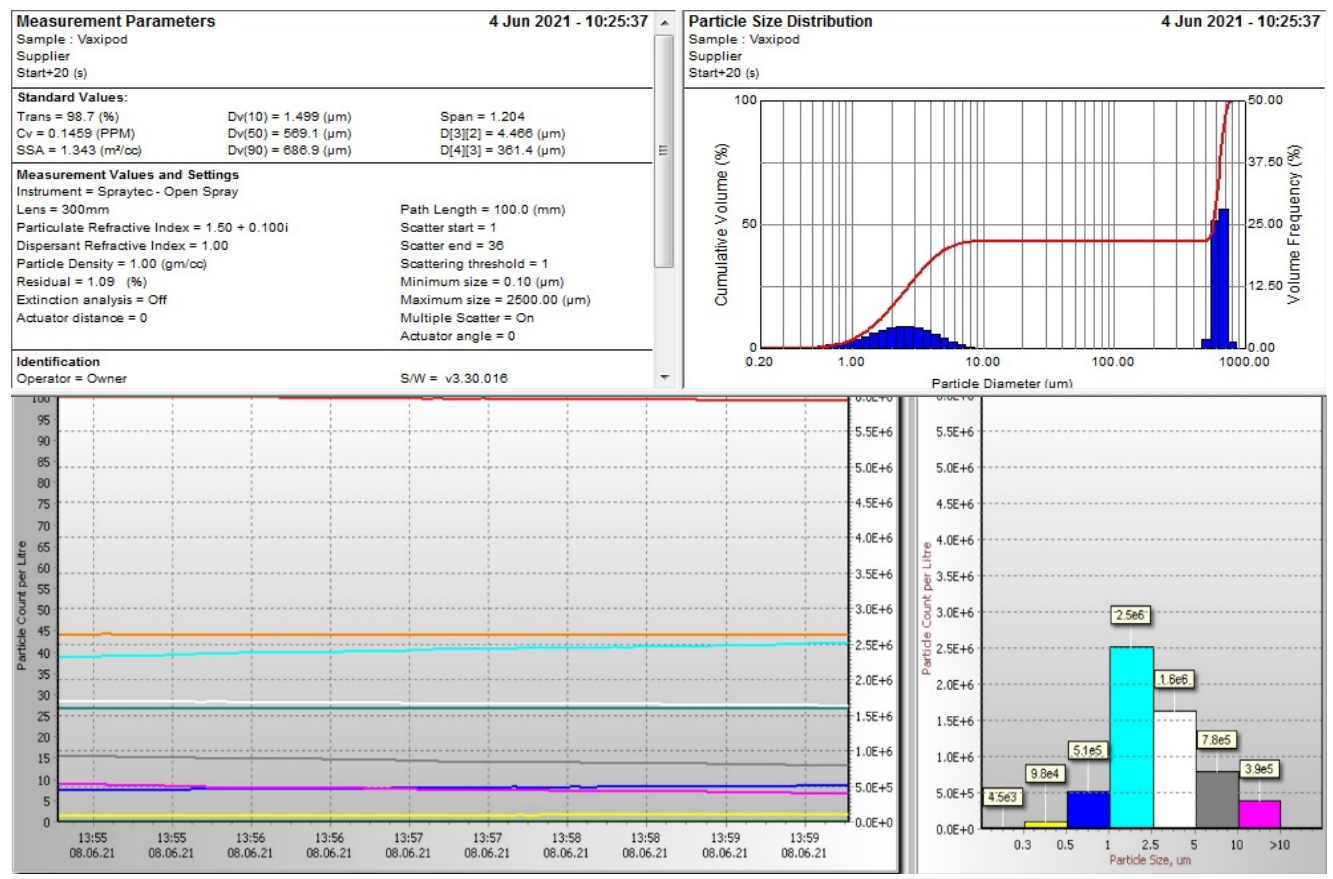

Figure 5. Size distribution of oils released by VaxiPod (Top figure was obtained by Sparytec, Malvern Panalytical, Malvern, UK; bottom figure was obtained by optical aerosol spectrometer, Model 4705, Aeronanotech, Moscow, Russia).

\subsection{Microbial Inactivation on Porous Filter Surface}

The results of microbial survival on the filter surface are shown in Figure 6. The inactivation of bacterial stains is presented respectively for E. coli and B. subtilis in Figure 6A,B. When no oil was supplied onto the fibre surface, only $38 \%$ of E. coli and $44 \%$ of B. subtilis were inactivated within the first $30 \mathrm{~min}$ of the experimental run, and after $60 \mathrm{~min}, 6 \%$ of E. coli and $27 \%$ of B. subtilis still remained viable. However, the presence of the oil in the environment drastically changed the behaviour of the survival curve, causing a substantial rate of bacteria decay and eliminating $97 \%$ of E. coli and $92 \%$ of B. subtilis within the first $30 \mathrm{~min}$ of each run. Only $0.5 \%$ and $4.0 \%$ remained viable after $60 \mathrm{~min}$ of operation for E. coli and B. subtilis, respectively, and no viable bacteria were detected on the filters after 120 min of operation.

The influence of oil presence was even more significant on the treatment of the airborne fungal strain of $A$. niger. When the oil was not supplied, the natural fungal decay was very slow, reaching only about $40 \%$ over the entire experimental run of $120 \mathrm{~min}$. Adding the oil via the VaxiPod enabled us to achieve significant fungal inactivation, even after $15 \mathrm{~min}$ of the process run (Figure 6C), reaching a value of $81 \%$. The rest of the experiment was not associated with such steep behaviour of the inactivation curve, reaching $89 \%$ after $30 \mathrm{~min}$ with further slow increase of the number, eliminating $92 \%$ of $A$. niger within the entire 120 min of operation.

The statistically significant inactivation of the viral strain of MS2 was achieved after 30 min of the oil atomiser operation with $40 \%$ remaining viable after that time. Only $2.3 \%$ of the bacteriophage remained viable after $2 \mathrm{~h}$ of the Vaxipod operation. Such outcome looks very promising as the inactivation curve obtained in absence of the oil does not look very impressive, reaching only about $50 \%$ of viral decay over two hours of the experimental run. 


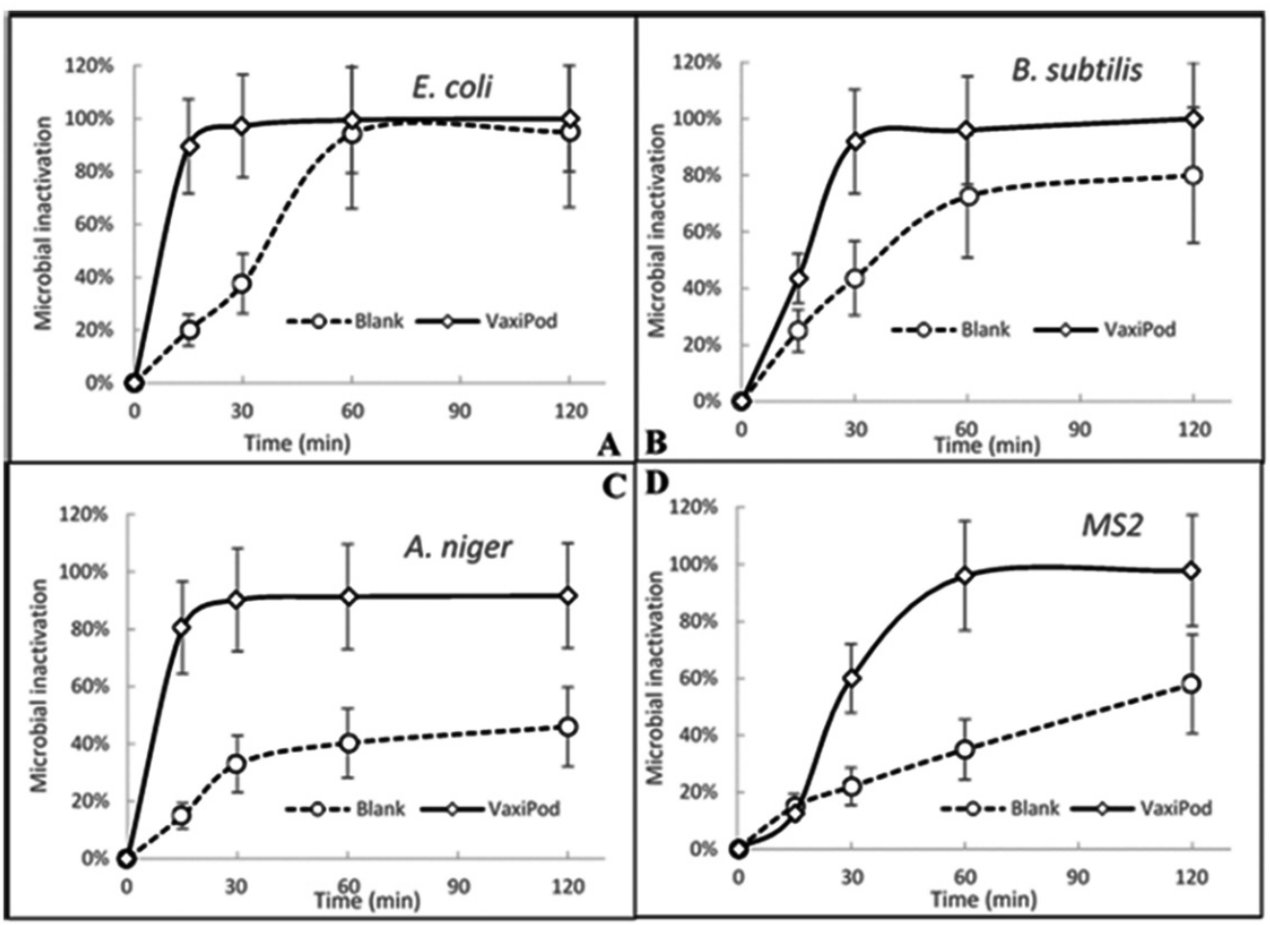

Figure 6. Microbial inactivation of (A) E. coli, (B) B. subtilis, (C) A. niger, (D) MS2 on the filter surface. Error bars represent STDEV of at least 3 experimental runs.

\subsection{Microbial Inactivation on Stainless Steel and Agar Surface}

The results of microbial inactivation on stainless steel discs and agar surface in the small enclosure are shown in Table 1. Overall, the oil mixture vapour was effective at reducing microbial load on the stainless steel surface compared with controls for the bacteria and bacteriophage after long exposure times. After $24 \mathrm{~h}$ of exposure to the aerosolised oil mix, the reductions of $71.4 \%$ and $94.6 \%$ were observed for $B$. subtilis and E. coli respectively, and the reduction of $74.3 \%$ for MS2 strain. On the other hand, the efficiency of fungal inactivation was much lower, reaching $41.4 \%$ after the same period of exposure. The situation was significantly improved after an additional $24 \mathrm{~h}$ of the experiment, reaching an almost perfect value for $E$. coli. The inactivation of microbial strains was slightly lower on the agar plates. As is seen, within $15 \mathrm{~h}$ of exposure to the dispersed oil blend, the highest reduction among all microorganisms was observed for E. coli, reaching $54.1 \%$. The corresponding inactivation was also observed in B. subtilis and A. niger, reaching a $46.9 \%$ and $22.9 \%$ reduction in viability, respectively. The oil mixture was less toxic to E. coli but more effective at inhibiting the growth of B. subtilis. During the following hours of the experimental run, all strains were inactivated, with the best result of $91.2 \%$ achieved for B. subtilis after two days of the experiment. Obviously, considering the entirely different nature of the plaque assay procedure, no results were obtained for MS2 on the agar plates.

Table 1. Mean reductions of microorganisms on stainless steel and agar surface when exposed to the oil mixture vapour in the small enclosure compared with controls that had not been exposed (deviations represent STD of at least three measurements).

\begin{tabular}{ccccccc}
\hline \multirow{2}{*}{ Organism } & \multicolumn{3}{c}{ Stainless Steel Discs } & \multicolumn{3}{c}{ Agar Plates } \\
\cline { 2 - 7 } & $\mathbf{1 5} \mathbf{h}$ & $\mathbf{1 ~ D a y}$ & 2 Days & $\mathbf{1 5 ~ h}$ & $\mathbf{1}$ Day & 2 Days \\
\hline E. coli & $84.8 \pm 9.5 \%$ & $94.6 \pm 6.7 \%$ & $>99.9 \pm 0.6 \%$ & $54.1 \pm 11.5 \%$ & $76.6 \pm 18.5 \%$ & $89.9 \pm 12.5 \%$ \\
\hline B. subtilis & $50.0 \pm 12.3 \%$ & $71.4 \pm 11.5 \%$ & $93.3 \pm 8.5 \%$ & $46.9 \pm 16.5 \%$ & $79.9 \pm 14.1 \%$ & $91.2 \pm 16.8 \%$ \\
\hline A. niger & $33.0 \pm 7.9 \%$ & $41.4 \pm 10.4 \%$ & $48.1 \pm 12.3 \%$ & $22.9 \pm 7.8 \%$ & $29.3 \pm 19.5 \%$ & $46.1 \pm 12.2 \%$ \\
\hline MS2 & $52.4 \pm 9.1 \%$ & $74.3 \pm 9.2 \%$ & $87.0 \pm 11.5 \%$ & & & \\
\hline
\end{tabular}




\subsection{Antimicrobial Activity against Biological Aerosols}

The results for microbial inactivation of E. coli, B. subtilis, A. niger and MS2 aerosols are shown in Figure 7. All results were normalised according to Equation (2). The error bars show standard deviation of at least three experimental runs.

The results for the microbial survival showed that almost $99.5 \%$ and $97 \%$ of microorganisms were inactivated within the first hour of the experimental run, with $92 \%$ and $85 \%$ inactivated during the first $15 \mathrm{~min}$ for E. coli and B. subtilis bacteria, respectively. There were no viable bacterial cells detected after two hours of the experiment.

Significant fungal inactivation was achieved after $30 \mathrm{~min}$ of treatment. $68 \%$ inactivation of A. niger fungal aerosol in the rotating chamber was achieved for the above time interval. The oil blend dispersed by the oil atomiser caused a substantial rate of microbial decay, eliminating $98 \%$ of $A$. niger within 120 min of each run.

It ought to be noticed that the results presented in Figure 7 were not only corrected by Equation (2). An additional very important correction was done to account for unavoidable gravitational losses occurring over two hours of the experimental runs [32]. As was shown in the referred publication, about $22 \%$ of microbial strains produced by the Collison Nebulizer and delivered to the Rotational Aerosol Chamber could settle after $2 \mathrm{~h}$ of the operation. Based on this number (confirmed in the current investigation), accounting for corresponding losses is extremely important as almost quoter of the aerosol could be affected. This issue is even more important for larger and "heavier" fungal aerosols, which were not used by Pyankov et al. in the referred publication. On this basis, in contrast with the previously shown graphs related to the bacterial and viral aerosols produced by the nebulizer [32] and respectively not copied to the current manuscript, the results obtained for the fungal particle are presented in Figure 8. As is seen, the settlement rate was substantially higher, with about $65 \%$ of particles naturally settling under action of gravity over $120 \mathrm{~min}$ of the experimental run. To make the figure more readable, the raw data is presented as a background (grey colour of the markers), whilst the trendline (moving average with period 10) data point markers are highlighted with black.

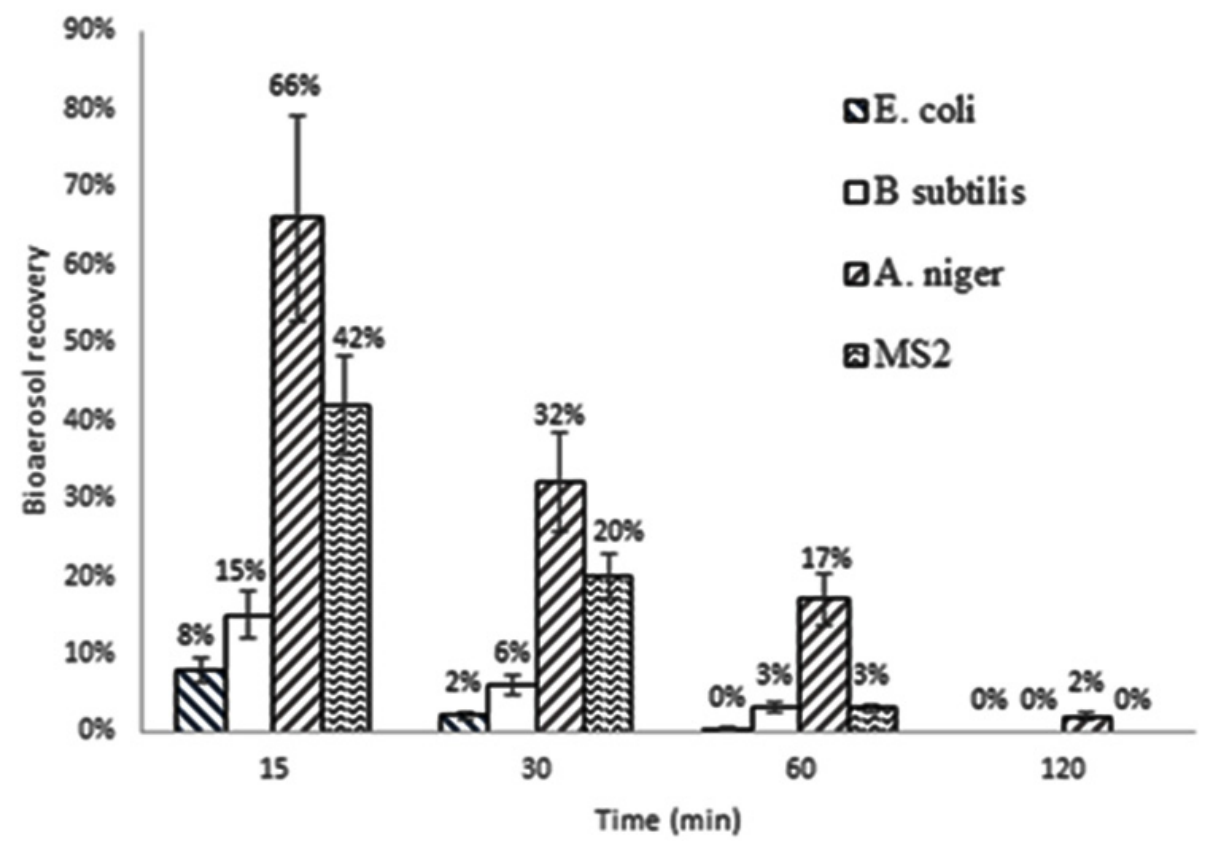

Figure 7. Microbial recovery of E. coli, B. subtilis, A. niger and MS2 aerosols treated with oil blend. 


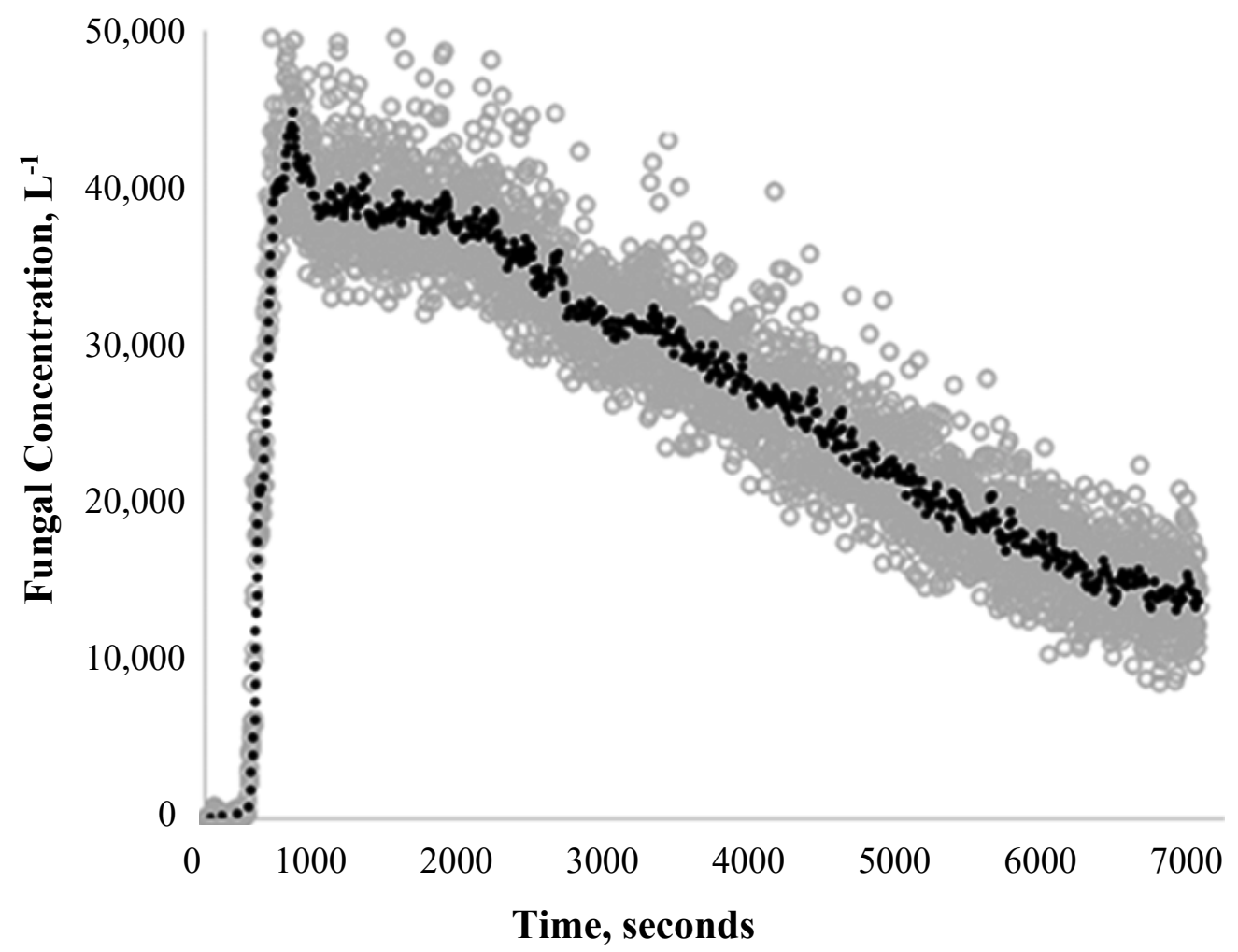

Figure 8. Natural settlement of $A$. niger fungal aerosol in the rotating aerosol chamber (raw data is shown in grey, and the trendline is presented in black).

\section{Discussion and Conclusions}

Essential oils added to the ambient air as vapour or aerosol are effective antimicrobial systems and their advantages over the use of EOs in their liquid phase include an increase in activity, use at lower concentrations and ability to be used in various environments. However, the spectrum of activities of EOs in the air against particular types of microorganism remains mainly undetermined [34]. This study assessed the efficacy of the essential oil blend Melaleuca alternifolia, Eucalyptus polybractea and Backhousia citriodora in respective ratios of 4.5:4.5:1.0, dispersed via the VaxiPod oil atomiser on two bacterial (E. coli and B. subtilis), one fungal (A. niger) and one viral (MS2 bacteriophage) strain commonly found in the atmospheric environment.

Inactivation of microbial aerosols settled on various surfaces is becoming exceptionally important research and technological question, considering that this is one of the most efficient transmission routes of a range of microbes, including COVID-19. Two surfaces used in this research demonstrated relatively similar results with slightly higher inactivation rates achieved on the stainless steel discs. It was expected that inactivation on the agar plates would be slower; however, the difference between the results is rather minor, dictating that the influence of the air environment dominates in the process, leaving the nature of the surface in second place. Of course, proper wiping of the surface with strong disinfectants would probably be more efficient; however, the proposed way of using VaxiPod would be much lower labour-consuming and could be a preferred option in many situations. The reported results look relatively similar to ones discussed in the Introduction section; however, a very important feature of the VaxiPod, related to its capability of $24 \mathrm{~h}$ of non-stop battery operation, enables much more flexible procedures of microbial inactivation to be used.

The results were also very encouraging when the contaminated filters were exposed to the oil blend vapour. As is seen, substantial rates of bacterial and viral inactivation eliminating $99.5 \%$ of E. coli, $95.0 \%$ of B. subtilis and $96 \%$ of MS2 on the filter surface were demonstrated within $60 \mathrm{~min}$ of the process run. Even for the very stress-resistant fungal strain of $A$. niger, the resulting decay of $91.7 \%$ was achieved within $2 \mathrm{~h}$ of operation. Such 
outcomes could be directly used in various scenarios. For example, the placement of the Vaxipod in the close vicinity to the air conditioning/ventilation air filters could firstly inactivate microorganisms on their surfaces, and then the vapour and uncaptured fine droplets delivered to the ambient air space can be used to disinfect microbial aerosols in their airborne form. Such placement would also be beneficial in achieving more even distribution of the oil vapour/droplets across substantially sized rooms, as powerful airflows created by ventilation/air conditioning systems would be used as compared to the low flowrate VaxiPod internal pump with much weaker capabilities in mixing with large-scale air flows.

In addition, a very useful feature of the VaxiPod, related to the capability of generating a second mode of aerosol particles with the diameter of a few hundred micrometres, would enable these particles to be effectively removed by even less efficient air filters used in residential and office air conditioning systems, ensuring the presence of a sufficient amount of the oil to be deposited on the filter to undertake the task.

The oil blend vapour dispersed by the Vaxipod demonstrated high efficiency in reducing airborne bacteria, fungi and viruses. The rapid inactivation of E. coli and B. subtilis with $92 \%$ and $85 \%$, respectively, was achieved within the first $15 \mathrm{~min}$, with only $0.4 \%$ and $3 \%$ remaining viable after two hours. Slightly lower inactivation of viral MS2 particle was observed over the first $30 \mathrm{~min}$ of the process run; however, the results became very comparable, reaching $97 \%$ of inactivation after $60 \mathrm{~min}$. To inactivate $98 \%$ of robust fungal aerosols, 120 min of the process run was required.

Of course, it is obvious that these results are rather indicative for common office/residential environments as these facilities are usually not isolated from the outdoor environment and fresh microbial particles will be continuously entering the space under disinfection. However, using more efficient air filters might significantly reduce such streams. Also, a very important issue could be related to the capability of inactivation of aerosols potentially released as a short-term burst, for example, from infected individual temporary attending a place (customer of the small boutique, visitor of a sales office, coffee shop client, etc.). In this case, a limited number of contagious bioaerosols could be quickly and effectively inactivated, minimising the possibility of long-term exposure of permanent staff.

Finally, it must also be noticed that essential oils emit many volatile organic compounds (VOCs), with some considered potentially hazardous [35]. As a result, using essential oils might be restricted for some groups of individuals.

Author Contributions: Conceptualization, E.M. and I.E.A.; Methodology, E.M. and I.E.A.; Formal Analysis, E.M. and I.E.A.; Investigation, E.M.; Resources, I.E.A.; Data Curation, E.M. and I.E.A.; Writing-Original Draft Preparation, E.M.; Writing-Review \& Editing, I.E.A.; Supervision, I.E.A.; Project Administration, I.E.A.; Funding Acquisition, I.E.A. All authors have read and agreed to the published version of the manuscript.

Funding: This research received no external funding.

Institutional Review Board Statement: Not applicable.

Informed Consent Statement: Not applicable.

Data Availability Statement: The datasets used and/or analysed during the current study are available from the corresponding author on reasonable request.

Conflicts of Interest: The authors declare no conflict of interest.

\section{References}

1. Otter, J.A.; Yezli, S.; Perl, T.M.; Barbut, F.; French, G.L. The role of 'no-touch' automated room disinfection systems in infection prevention and control. J. Hosp. Infect. 2013, 83, 1-13. [CrossRef] [PubMed]

2. Rai, M.; Zacchino, S.; Derita, M. (Eds.) Essential Oils and Nanotechnology for Treatment of Microbial Diseases; CRC Press: Boca Raton, FL, USA, 2017.

3. Taylor, R. Lemon myrtle, the essential oil. Rural Res. 1996, 172, 18-19. 
4. Carson, C.; Hammer, K.; Riley, T.V. Melaleuca alternifolia (Tea Tree) Oil: A Review of Antimicrobial and Other Medicinal Properties. Clin. Microbiol. Rev. 2006, 19, 50-62. [CrossRef] [PubMed]

5. Wilkinson, J.M.; Cavanagh, H.M.A. Antibacterial activity of essential oils from Australian native plants. Phytother. Res. 2005, 19, 643-646. [CrossRef] [PubMed]

6. Salari, M.H.; Amine, G.; Shirazi, M.H.; Hafezi, R.; Mohammadypour, M. Antibacterial effects of Eucalyptus globulus leaf extract on pathogenic bacteria isolated from specimens of patients with respiratory tract disorders. Clin. Microbiol. Infect. 2006, 12, 194-196. [CrossRef]

7. Hammer, K.A.; Carson, C.F.; Riley, T.V. Melaleuca alternifolia (tea tree) oil inhibits germ tube formation by Candida albicans. Med. Mycol. 2000, 38, 355-362. [CrossRef] [PubMed]

8. Oliva, B.; Piccirilli, E.; Ceddia, T.; Pontieri, E.; Aureli, P.; Ferrini, A. Antimycotic activity of Melaleuca alternifolia essential oil and its major components. Lett. Appl. Microbiol. 2003, 37, 185-187. [CrossRef] [PubMed]

9. Schnitzler, P.; Schön, K.; Reichling, J. Antiviral activity of Australian tea tree oil and eucalyptus oil against herpes simplex virus in cell culture. Die Pharm. 2001, 56, 343-347.

10. Garozzo, A.; Timpanaro, R.; Stivala, A.; Bisignano, G.; Castro, A. Activity of Melaleuca alternifolia (tea tree) oil on Influenza virus A/PR/8: Study on the mechanism of action. Antivir. Res. 2011, 89, 83-88. [CrossRef]

11. May, J.; Chan, C.H.; King, A.; Williams, L.; French, G.L. Time-kill studies of tea tree oils on clinical isolates. J. Antimicrob. Chemother. 2000, 45, 639-643. [CrossRef] [PubMed]

12. Barbosa, L.C.A.; Filomeno, C.A.; Teixeira, R.R. Chemical Variability and Biological Activities of Eucalyptus spp. Essential Oils. Molecules 2016, 21, 1671. [CrossRef]

13. Pyankov, O.; Usachev, E.V.; Pyankova, O.; Agranovski, I.E. Inactivation of Airborne Influenza Virus by Tea Tree and Eucalyptus Oils. Aerosol Sci. Technol. 2012, 46, 1295-1302. [CrossRef]

14. Usachev, E.V.; Pyankov, O.; Usacheva, O.V.; Agranovski, I.E. Antiviral activity of tea tree and eucalyptus oil aerosol and vapour. J. Aerosol Sci. 2013, 59, 22-30. [CrossRef]

15. Southwell, I.A.; Russell, M.; Smith, R.L.; Archer, D.W. Backhousia citriodora F. Muell. (Myrtaceae), A Superior Source of Citral. J. Essent. Oil Res. 2000, 12, 735-741. [CrossRef]

16. Hayes, A.; Markovic, B. Toxicity of Australian essential oil Backhousia citriodora (Lemon myrtle). Part 1. Antimicrobial activity and in vitro cytotoxicity. Food Chem. Toxicol. 2002, 40, 535-543. [CrossRef]

17. Wilkinson, J.M.; Hipwell, M.; Ryan, A.T.; Cavanagh, H.M.A. Bioactivity of Backhousia citriodora: Antibacterial and Antifungal Activity. J. Agric. Food Chem. 2003, 51, 76-81. [CrossRef] [PubMed]

18. Tian, F.; Lee, S.Y.; Chun, H.S. Comparison of the Antifungal and Antiaflatoxigenic Potential of Liquid and Vapor Phase of Thymus vulgaris Essential Oil against Aspergillus flavus. J. Food Prot. 2019, 82, 2044-2048. [CrossRef] [PubMed]

19. Inouye, S.; Takizawa, T.; Yamaguchi, H. Antibacterial activity of essential oils and their major constituents against respiratory tract pathogens by gaseous contact. J. Antimicrob. Chemother. 2001, 47, 565-573. [CrossRef]

20. Bassolé, I.H.N.; Juliani, H.R. Essential Oils in Combination and Their Antimicrobial Properties. Molecules 2012, 17, 3989-4006. [CrossRef] [PubMed]

21. Doran, A.L.; Morden, W.E.; Dunn, K.; Edwards-Jones, V. Vapour-phase activities of essential oils against antibiotic sensitive and resistant bacteria including MRSA. Lett. Appl. Microbiol. 2009, 48, 387-392. [CrossRef] [PubMed]

22. Gaunt, L.; Higgins, S.; Hughes, J. Interaction of air ions and bactericidal vapours to control micro-organisms. J. Appl. Microbiol. 2005, 99, 1324-1329. [CrossRef] [PubMed]

23. Chao, S.C.; Young, D.G.; Oberg, C.J. Effect of a Diffused Essential Oil Blend on Bacterial Bioaerosols. J. Essent. Oil Res. 1998, 10, 517-523. [CrossRef]

24. Lanzerstorfer, A.; Hackl, M.; Schlömer, M.; Rest, B.; Deutsch-Grasl, E.; Lanzerstorfer, C. The influence of air-dispersed essential oils from lemon (Citrus limon) and silver fir (Abies alba) on airborne bacteria and fungi in hospital rooms. J. Environ. Sci. Health Part A 2019, 54, 256-260. [CrossRef]

25. Krist, S.; Sato, K.; Glasl, S.; Hoeferl, M.; Saukel, J. Antimicrobial effect of vapours of terpineol,(R)-(-)-linalool, carvacrol,(S)-(-)perillaldehyde and 1, 8-cineole on airborne microbes using a room diffuser. Flavor Frag. J. 2008, 23, 353-356. [CrossRef]

26. Gelmini, F.; Belotti, L.; Vecchi, S.; Testa, C.; Beretta, G. Air dispersed essential oils combined with standard sanitization procedures for environmental microbiota control in nosocomial hospitalization rooms. Complement. Ther. Med. 2016, 25, 113-119. [CrossRef]

27. Diaz-Alonso, J.; Bernardos, A.; Regidor-Ros, J.L.; Martinez-Manez, R.; Bosch-Roig, P. Innovative use of essential oil cold diffusion system for improving air quality on indoor cultural heritage spaces. Int. Biodeterior. Biodegrad. 2021, 162, 105251. [CrossRef]

28. Gismondi, A.; Di Marco, G.; Redi, E.L.; Ferrucci, L.; Cantonetti, M.; Canini, A. The antimicrobial activity of Lavandula angustifolia Mill. essential oil against Staphylococcus species in a hospital environment. J. Herb. Med. 2021, 26, 100426. [CrossRef]

29. Adams, M.H. Bacteriophages; Interscience Publishers: New York, NY, USA, 1959.

30. Scheermeyer, E.; Agranovski, I.E. Design and evaluation of a new device for fungal spore aerosolization for laboratory applications. J. Aerosol Sci. 2009, 40, 879-889. [CrossRef]

31. Agranovski, I.; Safatov, A.; Borodulin, A.; Pyankov, O.; Petrishchenko, V.; Sergeev, A.; Agranovski, V.; Grinshpun, S. New personal sampler for viable airborne viruses: Feasibility study. J. Aerosol Sci. 2005, 36, 609-617. [CrossRef]

32. Pyankov, O.; Pyankova, O.G.; Agranovski, I.E. Inactivation of airborne influenza virus in the ambient air. J. Aerosol Sci. 2012, 53, 21-28. [CrossRef] 
33. Agranovski, I.E.; Braddock, R.D.; Myojo, T. Removal of Aerosols by Bubbling through Porous Media. Aerosol Sci. Technol. 1999, 31, 249-257. [CrossRef]

34. Laird, K.; Phillips, C. Vapour phase: A potential future use for essential oils as antimicrobials? Lett. Appl. Microbiol. 2012, 54, 169-174. [CrossRef] [PubMed]

35. Nematollahi, N.; Weinberg, J.L.; Flattery, J.; Goodman, N.; Kolev, S.D.; Steinemann, A. Volatile chemical emissions from essential oils with therapeutic claims. Air Qual. Atmos. Health 2020, 14, 365-369. [CrossRef] 